\title{
Three-dimensional modeling and assessment of cardiac adipose tissue distribution
}

\author{
Jon D. Klingensmith ${ }^{1}$, Saygin Sop ${ }^{2}$, Mete Naz $^{2}$, Maria Fernandez-del-Valle ${ }^{3}$, H. Felix Lee ${ }^{2}$ \\ ${ }^{1}$ Department of Electrical and Computer Engineering, Southern Illinois University Edwardsville, Edwardsville, IL, USA \\ ${ }^{2}$ Department of Mechanical and Industrial Engineering, Southern Illinois University Edwardsville, Edwardsville, IL, USA \\ ${ }^{3}$ Department of Applied Health, Southern Illinois University Edwardsville, Edwardsville, IL, USA
}

Correspondence: Jon D. Klingensmith, Ph.D. E-mail address: jokling@siue.edu

Received: December 6, 2017

Accepted: December 29, 2017

Online Published: January 15, 2018

DOI: $10.5430 / j b g c . v 8 n 1 p 14$

URL: http://dx.doi.org/10.5430/jbgc.v8n1p14

\begin{abstract}
Objective: The layer of fat that accumulates around the heart, called cardiac adipose tissue (CAT), can influence the development of coronary disease and is indicative of cardiovascular risk. While volumetric assessment of magnetic resonance imaging (MRI) can quantify CAT, volume alone gives no information about its distribution across the myocardial surface, which may be an important factor in risk assessment. In this study, a three-dimensional (3D) modeling technique is developed and used to quantify the distribution of the CAT across the surface of the heart.

Methods: Dixon MRI scans, which produce a registered 3D set of fat-only and water-only images, were acquired in 10 subjects for a study on exercise intervention. A previously developed segmentation algorithm was used to identify the heart and CAT. Extracted contours were used to build 3D models. Procrustes analysis was used to register the heart models and an iterative closest point algorithm was used to register and align the CAT models for calculation of CAT thickness. Rays were cast in directions specified by a spherical parameterization of elevation and azimuthal angles, and intersections of the ray with the CAT surface were used to calculate the thickness at each location. To evaluate the effects of the spherical parameterization on the thickness estimates, a set of synthetic models were created with increasing major-to-minor axis ratios.
\end{abstract}

Results: Based on the experimental data from the synthetic models, the average error in CAT thickness ranged from $1.25 \%$ to $17.3 \%$ for increasing major-to-minor axis ratio.

Conclusions: A process was developed, based on Dixon MRI data, to provide 3D models of the myocardial surface and the cardiac fat. The models can be used in future segmentation algorithm development and for studies on changes in cardiac fat as a result of various interventions.

\section{Key Words:}

Epicardial adipose tissue, Cardiac fat, Three-dimensional modeling, Dixon MRI

\section{Introduction}

Obesity is associated with significant risk of developing cardiovascular disease ${ }^{[1,2]}$. Excess adipose tissue in the human body is divided into two main categories: subcutaneous, located below the skin, and visceral, located in the abdominal cavity and surrounding the internal organs. One of these ectopic fat depots is associated with the heart and develops between the myocardium and visceral pericardium. This fat deposit is known as epicardial adipose tissue (EAT). There 
can also be additional adipose tissue between the visceral and parietal pericardium; this fat is typically referred to as the paracardial adipose tissue (PAT). We subsequently refer to the combined EAT and PAT as cardiac adipose tissue (CAT). The CAT is metabolically dynamic and secretes active cytokines that can influence coronary arterial wall homeostasis and the development of coronary artery disease (CAD). More specifically, there is no fascia between the myocardium and the EAT $^{[3]}$. The EAT is therefore in direct contact with the coronary vasculature and can maintain an active inflammation process and the progression of $\mathrm{CAD}^{[4]}$.

Studies have shown that there is a strong relationship between $\mathrm{CAT}$ volume and $\mathrm{CAD}^{[4,5]}$. CAT volume correlates directly with both the presence of CAD and severity of stenosis, but the relationship also extends beyond the volume of plaque alone. Soft, smaller heterogeneous plaques ("mixed" plaques), consisting of many different tissue types, but not blocking the entire lumen, are more metabolically active and therefore more dangerous. Increased CAT volume, measured by cardiac computed tomography (CT), is associated with development of the most dangerous types of plaques ${ }^{[5]}$. In addition, increased CAT volume also suggests increased likelihood of the presence of disease specifically in the left anterior descending (LAD) coronary artery ${ }^{[6]}$. The LAD is particularly significant because it perfuses large areas of myocardium around the left ventricle. Because of the strong link between CAT and cardiovascular disease, it is important to develop non-invasive, in-vivo quantification techniques.

Both cardiac CT and cardiac magnetic resonance imaging (MRI) have proven useful in quantifying CAT ${ }^{[5-11]}$. MRI has emerged as a highly effective imaging modality for identifying whole body fat and ectopic fat depots "[12]. "Dixon" technique sequences create a registered set of in-phase (IP) and out-of-phase (OOP) image volumes and can be used to highlight adipose tissue by producing fat-only and water-only images ${ }^{[13]}$. The three-dimensional (3D) data sets created by MRI are rich with information, but most of the work to date has focused on quantifying overall volume and has not assessed the distribution of the CAT ${ }^{[9,11,14]}$. In addition, automated and semi-automated algorithms have been developed to assess CAT volume from cardiac MRI, aiding in the cumbersome process of analyzing the large number of images involved with the $3 \mathrm{D}$ scans ${ }^{[15,16]}$. In a previous study, our group has also worked to develop and test an automated segmentation algorithm to identify the CAT in 3D Dixon MRI images ${ }^{[17]}$. In addition, the shape of cardiac structures has been investigated extensively. Statistical shape models (SSMs) have been built to facilitate quantification and non-invasive measurement of cardiac structures and related functional parameters ${ }^{[18-22]}$. However, these studies have focused on atria and ventricles. Little work exists to investigate and model the CAT, including its quantity and distribution. The paracrine activity of the CAT is local in nature and the proximity of this adipose tissue to the coronary arteries may be an important aspect of cardiovascular risk dynamics. Therefore, the objective of this study was to develop a three-dimensional model for assessment of CAT distribution.

\section{Methods}

\subsection{Image Data Acquisition}

The models were created using MRI scans of 10 female subjects enrolled in a study of exercise intervention on excess adiposity. Volunteer subjects were healthy, between the ages of 18 and 30 years old, and had BMI values between 30 and $39.99 \mathrm{~kg} / \mathrm{m}^{2}$ (Obesity Class I and II). Subjects were excluded if they had one or more of the following six characteristics: (1) at risk for cardiovascular events, (2) pregnant, (3) were on medications affecting endocrine or cardiovascular function, (4) have high blood pressure, (5) are already engaging in strength-training more than twice per week or any type of moderate-high intensity exercise program, and (6) are smokers. The study was approved by the local Internal Review Board and informed consent was agreed upon by each volunteer subject.

For the MRI acquisition, coils were used to acquire data from both the thoracic and abdominal cavities using a 3.0 T Skyra system (Siemens, Munich, Germany) and a multi-echo T1-weighted Volumetric Interpolated Breath-hold Examination (VIBE) 2-point Dixon imaging sequence ${ }^{[23,24]}$ with a 20 -second breath hold $\left(\mathrm{TR} / \mathrm{TE}=3.97 / 1.23 \mathrm{~ms}\right.$, flip angle $\left.=9^{\circ}\right)$. During image acquisition, subjects lay in a supine position, arms extended above their head, and support pillow beneath their knees. Image volumes with 120 slices ( $2.5 \mathrm{~cm}$ thick with $20 \%$ gap) and $320 \times 260$ pixels in each slice were acquired over a field-of-view (FOV) of $45 \mathrm{~cm}$. Based on this geometry, the effective voxel size was $1.40625 \times 1.40625 \times 3.0 \mathrm{~mm}$. The IP and OOP registered image volumes were used by the scanner software to reconstruct the fat-only and water-only images.

\subsection{Image Analysis and Modeling}

The automatic segmentation software used in this study for identification of the heart was developed and implemented in MATLAB (MathWorks, Natick, MA). Briefly, the MRI volumes were analyzed in three main steps: (1) rectification of the volumes from the 2 coils, (2) labeling of anatomical landmarks to facilitate segmentation, and (3) a sequential segmentation of anatomical structures and ectopic fat depots. Details of the algorithm are described in previous work ${ }^{[17]}$. It 
provides segmentation of both the heart and the cardiac fat. The labeled images from the segmentation algorithm were further post-processed prior to extracting the heart and fat contours. An additional morphological opening and closing operation, using a circular structure element of radius 3, was used to smooth the boundaries of the shape. Then, contours were extracted from the labeled binary images using the Moore-Neighbor tracing algorithm ${ }^{[25]}$ and saved for use in the modeling process. An example image slice with the labeled heart pixels and extracted heart contours is shown in Figure 1. Corresponding CAT pixels and extracted CAT contours are shown in Figure 2.

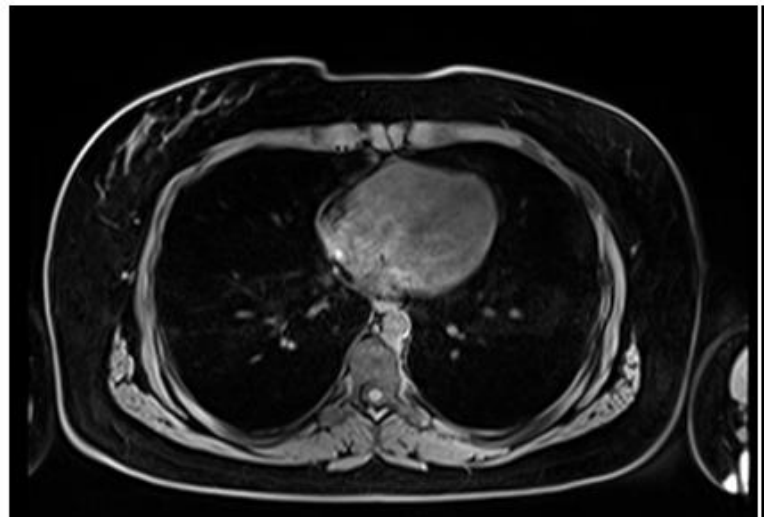

(a)

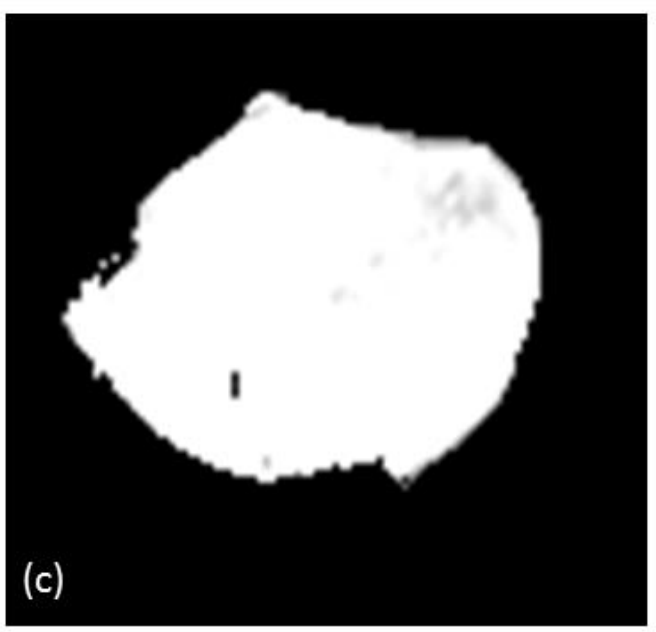

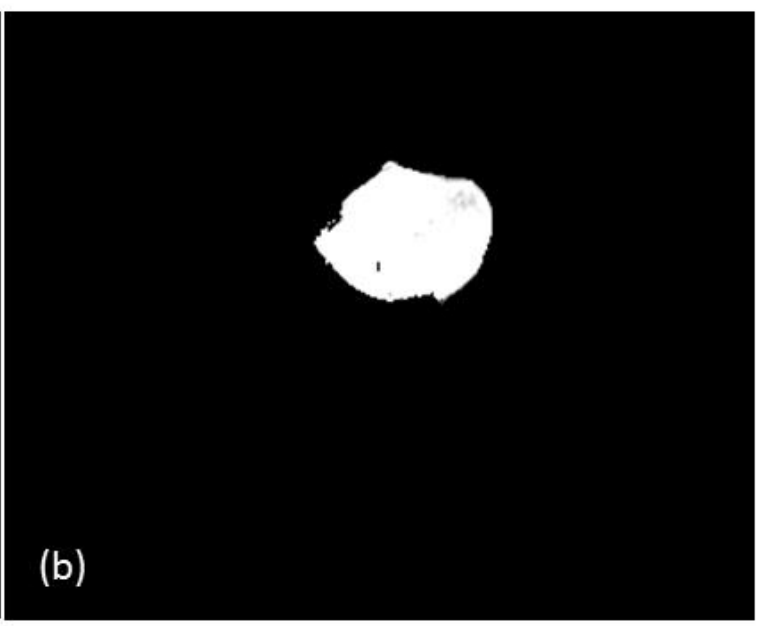

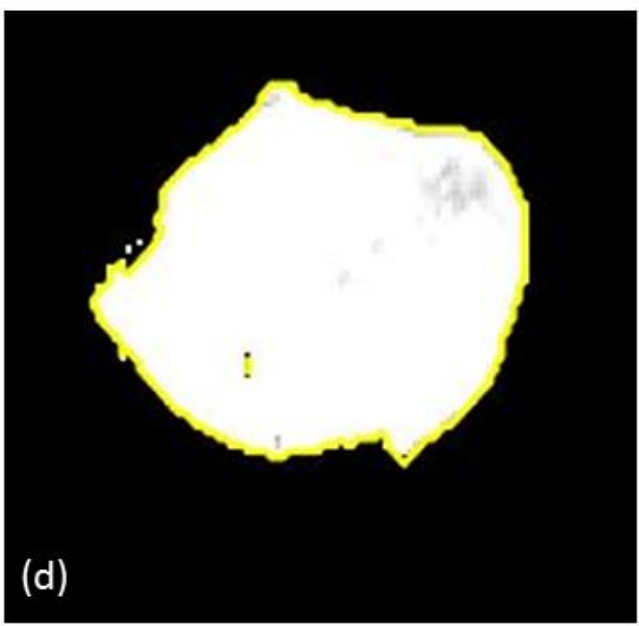

Figure 1. Example heart segmentation

Example Dixon MRI (water-only) image (a) and segmented heart (b). A close-up of the segmented heart is shown in (c) and extracted heart contours (in yellow) are illustrated in (d). 

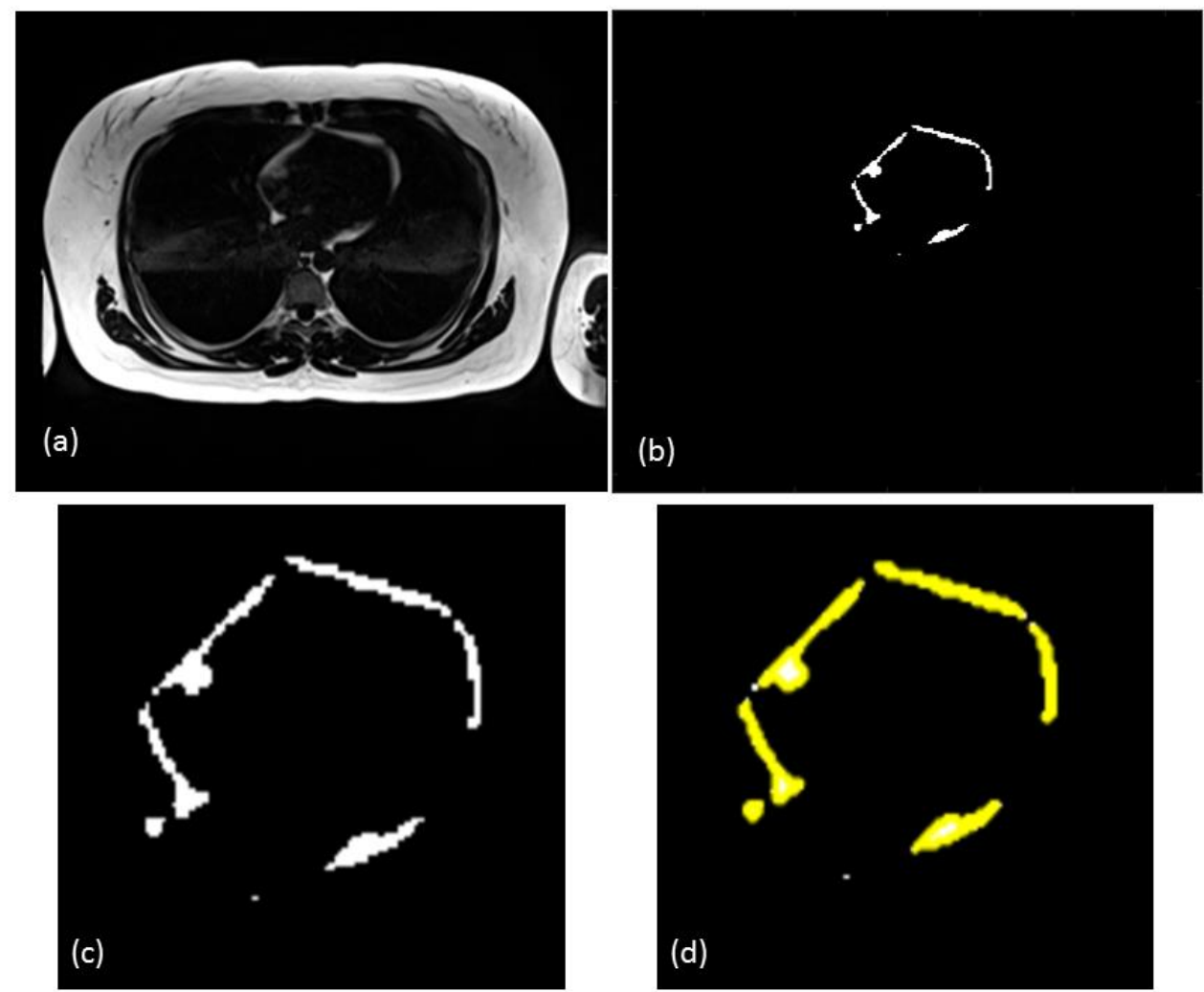

Figure 2. Example CAT segmentation

Example Dixon MRI (fat-only) image (a) and segmented cardiac adipose tissue (CAT) (b). A close-up of the segmented CAT is shown in (c) and extracted CAT contours (in yellow) are illustrated in $(d)$.

The contours extracted from the segmented heart were imported into NX10 (Siemens PLM Software, Plano, TX) and used to build the 3D heart models. First, a spline was fit to the in-plane contours, approximating the data points, but smoothing the shape and providing structure. The tradeoff between the closeness of fit to the data points and the smoothness of the spline contour was adjusted manually for each set of contours from each image slice. Through-plane guide curves were also extracted from the original data points (every 15 degrees) and used to give the model structure along the direction of the long axis. The process was repeated using the cardiac fat contours to create the model of the adipose tissue. An example interim model with guide curves and completed model with both heart surface and fat layer is shown in Figure 3.
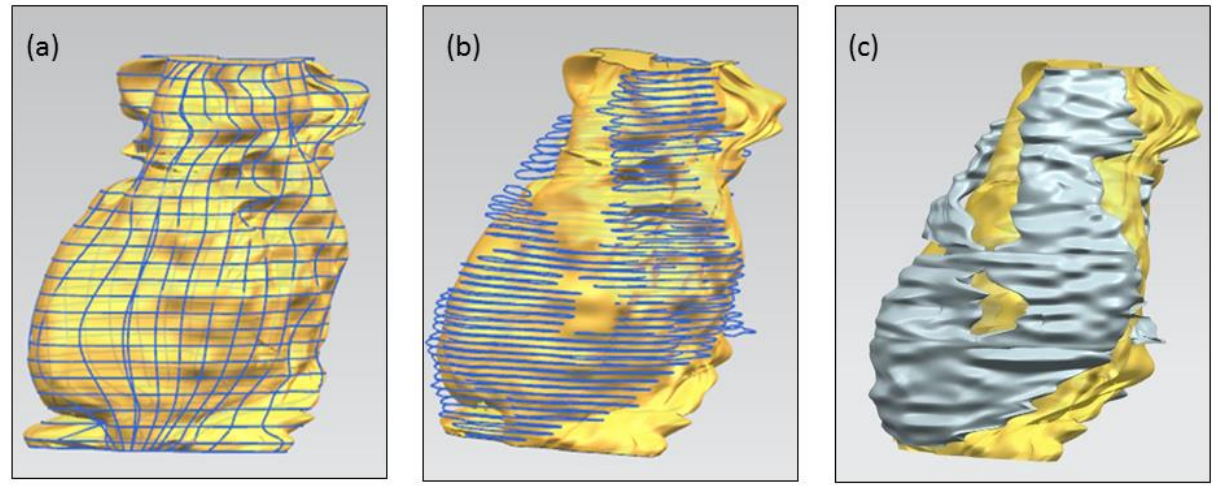

Figure 3. Example model with guide curves illustrated

The heart surface with guide curves is shown in (a). The fat model, rendered as raw splines, is shown in (b). The fat model, rendered as a surface, is shown in (c). 


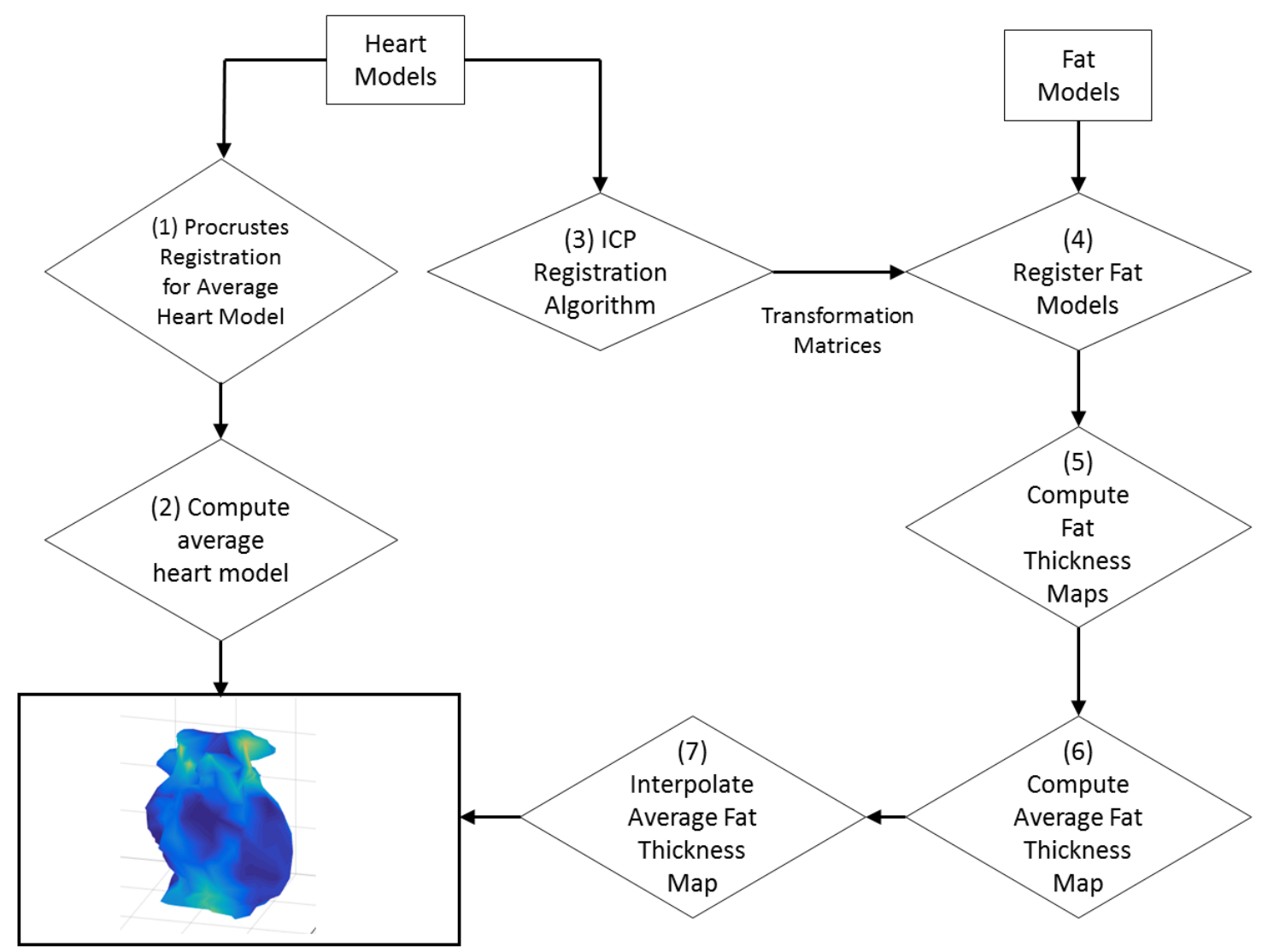

Figure 4. Flow chart of algorithm

The left-side path is used to create the average heart surface and the right-side path is used to calculate the fat thickness maps. The two are merged to create the overall surface model with mean fat thickness applied.

The algorithm for analyzing the quantity and distribution of cardiac fat is shown in Figure 4 and uses the individual heart and fat models as inputs. The two major branches of the algorithm are indicated in the flow chart: a formulation of the average heart surface model and the computation of the average fat thickness across the surface of the heart for each data set. The average heart model was created using Procrustes analysis (step (1) in Figure 4 flow chart), which determines the optimal set of translation, scaling, and rotation transformations to align a set of landmarks or points. The heart models were represented in polygonal format with a set of vertices and triangular faces. Given $\boldsymbol{X}$ as the set of vertices from one model and $\boldsymbol{Y}$ as the set of vertices from another, Procrustes analysis aims to minimize the following in a least-squares sense where $\|\boldsymbol{X}\|$ is the Euclidean norm of $\boldsymbol{X}$ :

$$
D(X, Y)=\|Y-\alpha X \beta+\delta\|^{2}
$$

$\boldsymbol{X}$ and $\boldsymbol{Y}$ are $3 \times k$ matrices (for three-dimensional Procrustes analysis) where $k$ is the number of vertices. $\alpha$ is the scaling component (a scalar), $\beta$ is the reflection and rotation component (a $k \mathrm{x} k$ matrix), and $\delta$ is the translation component (a $3 \mathrm{x}$ $k$ matrix). Each of these, $\alpha, \beta$, and $\delta$ are solved for via Procrustes analysis ${ }^{[26]}$. Once the individual matrices for each model $(\alpha, \beta$, and $\delta)$ were computed, they were applied to the heart models to scale, rotate, and translate them for alignment. An example set of heart model vertices, before and after Procrustes registration, are shown in Figure 5. After each of the heart models were registered, the average surface was computed by taking the mean location of each of the corresponding vertices from the heart models, as indicated by step (2) in the flow chart. 

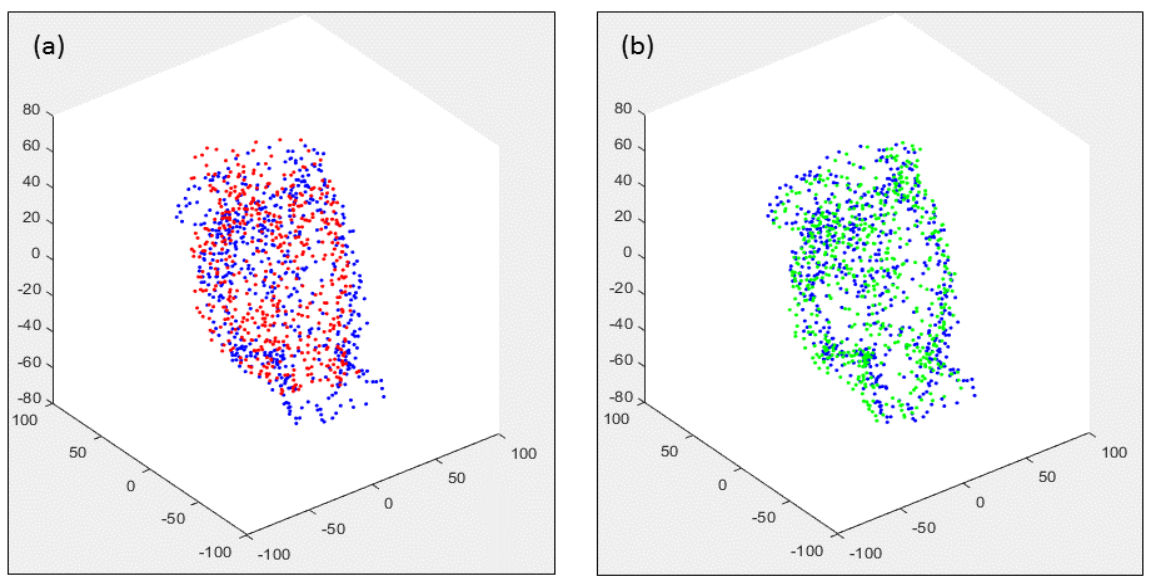

Figure 5. Example heart vertices from Procrustes registration

(a) shows the reference points (in blue) and data points (in red) before registration. (b) shows the reference points (in blue) and data points (in green) after registration.

The other branch of the process involved the computation of the distribution of cardiac fat thickness across the surface of the heart for each individual model created. For this step, an iterative closest point (ICP) registration algorithm ${ }^{[27]}$ was used to determine the appropriate rotation and translation matrices. Traditional ICP algorithms involve a similar construct as is shown in Equation (1), but without the scaling component. In this algorithm, the optimal rotation and translation matrices are determined using an iterative approach where corresponding points between the reference model and the data model are determined using a distance metric for each iteration. The difference between the reference points and the model points, as modified by the proposed rotation and translation matrices, is minimized iteratively in a least-squared sense until the convergence criteria are met or a maximum number of iterations is reached. The specific algorithm used here, proposed by Bergstrom and Edlund, is modified to limit the influence of outliers by re-weighting the least-squares problem for each iteration $^{[27]}$. This step is represented in the flow chart (Figure 4) in step (3). The translation and rotation matrices determined for each individual model (based on the same reference model for each) were applied to the vertices of the fat model to align them and create a common frame of reference for fat thickness computation, denoted by step (4) in the flow chart. An example set of vertices from aligned fat models are shown in Figure 6a.
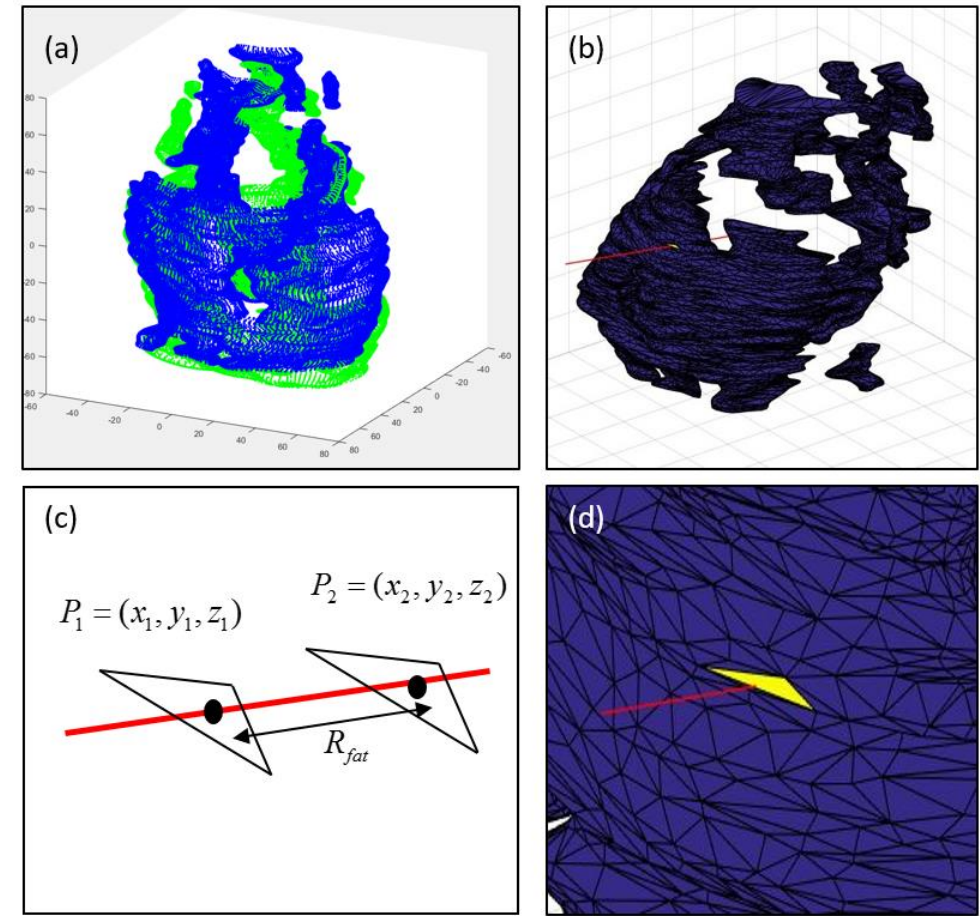

Figure 6. Fat thickness calculation 
Two registered sets of fat model points are shown in (a). (b) illustrates the ray (in red) that is cast from the center of the model and used for fat thickness computation. The triangle that the ray intersects with is highlighted in yellow. (c) illustrates an example of 2 intersection points that would be used for the thickness calculation along the ray and (d) is a close-up of the intersected triangle.

Once registered to the common frame of reference, the thickness of fat was calculated across the surface of the heart (step (5) in the flow chart). The sampling strategy for the locations of fat thickness was based on a spherical parameterization $(\theta, \varphi)$ where $\theta$ represents the azimuthal angle (angle from positive $x$-axis) and $\varphi$ represents the elevation angle (angle from the $x-y$ plane). The points on the fat model were translated so that the origin of the $3 \mathrm{D}$ coordinate system was at the centroid of the model points. Then, for each pair of angles for $\theta$ from $90^{\circ}$ to $-90^{\circ}$ and for $\varphi$ from $0^{\circ}$ to $360^{\circ}$, a ray was cast from the origin in the direction indicated by the pair of angles. To compute the fat thickness map for the $(\theta, \varphi)$ parameterization, intersections of the ray with the fat model were calculated. The models were represented as a set of vertices and triangular faces and the required intersection points were determined using a ray/triangle intersection algorithm ${ }^{[28]}$. The ray can be represented as shown in Equation (2)

$$
R(t)=O+t D
$$

where $R$ is the set of $(x, y, z)$ coordinates of the ray vector, $O$ is the origin, $D$ is the set of $(x, y, z)$ coordinates of the ray direction, and $t$ is the distance along the ray. In the formulation used here, the directions, $D$, of each ray to use for the parameterization map were determined by converting from spherical to Cartesian coordinates, as shown in Equations (3), (4), and (5)

$$
\begin{aligned}
& x=r \cos (\varphi) \cos (\theta) \\
& y=r \cos (\varphi) \sin (\theta) \\
& z=r \sin (\varphi)
\end{aligned}
$$

where $r$ is chosen to ensure that the Cartesian point is sufficiently distant from the origin to be outside potential model surface intersections. Locations across the planar surface of each individual triangle are given by Equation (6)

$$
T(u, v)=(1-u-v) V_{1}+u V_{2}+v V_{3}
$$

where $u$ and $v$ are the barycentric coordinates and $V_{1}, V_{2}$, and $V_{3}$ are the three vertex locations of the triangle. The intersection between the ray and any given triangle, therefore, can be determined by setting Equations (2) and (6) equal to each other, rearranging the terms, and then solving the system of linear equations ${ }^{[28]}$.

The fat deposits near the base of the heart and the ostia of the right coronary artery (RCA) and the left main coronary artery create non-trivial surface topologies in the fat models. They are not "connected" surfaces, meaning that there may be some points on the surface model that are not connected by a path along the surface to other points on the model. In other words, there could be multiple "objects" associated with the model surface. The fat thickness at any given $(\theta, \varphi)$ location was calculated as the total, accumulated thickness of adipose tissue that the ray traveled through as it passed through the model. This concept is illustrated in Figure $6 \mathrm{~b}$ and $6 \mathrm{~d}$, where the intersected triangles on the surface are shown in yellow. Ray/triangle intersections, in Cartesian coordinates, were taken in pairs to calculate the adipose tissue thickness along that particular angle defined by the $(\theta, \varphi)$ location. The Cartesian coordinates of each point were used to compute adipose tissue thickness for each pair of intersections, as shown in Figure 6c and Equation (7).

$$
R_{f a t}=\sqrt{x_{2}^{2}+y_{2}^{2}+z_{2}^{2}}-\sqrt{x_{1}^{2}+y_{1}^{2}+z_{1}^{2}}
$$

The adipose tissue thickness $R_{f a t}$, in $\mathrm{mm}$, was recorded for the fat map based on the $(\theta, \varphi)$ spherical parameterization.

This process was repeated for all 10 models, and the resulting maps were used to compute an average fat thickness, as indicated by step (6) in the flow chart in Figure 4. The last step, indicated by step (7) in the flow chart, involved applying the average fat thickness values to appropriate locations on the average heart model. The mapping of locations of the average fat thickness to the average heart surface model was performed using the $(\theta, \varphi)$ spherical parameterization. For each vertex on the model, the $\mathrm{x}, \mathrm{y}$, and $\mathrm{z}$ Cartesian coordinates were converted to spherical coordinates using equations (8) and (9) as shown 


$$
\begin{gathered}
\theta=\arctan \left(\frac{y}{x}\right) \\
\varphi=\arctan \left(\frac{z}{\sqrt{\left(x^{2}+y^{2}\right)}}\right)
\end{gathered}
$$

This $(\theta, \varphi)$ coordinate pair was used as in index into the average fat map and the closest value was used as the fat thickness for that location on the heart surface. Bilinear interpolation was used to determine fat thickness values for shading of the model between vertices.

\subsection{Synthetic Model Experiments}

To evaluate the effects of the spherical parameterization on the thickness estimates, a set of synthetic models were created. The first was spherical, but the remaining models were ellipsoids and created with a major axis increment of $10 \mathrm{~mm}$. The minor axes were held constant at $110 \mathrm{~mm}$.

\begin{tabular}{|c|c|c|c|c|c|}
\hline Model & $\begin{array}{l}\text { Inside } \\
\text { Minor }\end{array}$ & $\begin{array}{c}\text { Outside } \\
\text { Minor }\end{array}$ & $\begin{array}{l}\text { Inside } \\
\text { Major }\end{array}$ & $\begin{array}{c}\text { Outside } \\
\text { Major }\end{array}$ & Thickness \\
\hline Synthetic_1 & 102 & 110 & 92 & 100 & 8 \\
\hline Synthetic_2 & 102 & 110 & 102 & 110 & 8 \\
\hline Synthetic_3 & 102 & 110 & 112 & 120 & 8 \\
\hline Synthetic_4 & 102 & 110 & 122 & 130 & 8 \\
\hline Synthetic_5 & 102 & 110 & 132 & 140 & 8 \\
\hline Synthetic_6 & 102 & 110 & 142 & 150 & 8 \\
\hline Synthetic_7 & 102 & 110 & 152 & 160 & 8 \\
\hline Synthetic_8 & 102 & 110 & 162 & 170 & 8 \\
\hline Synthetic_9 & 102 & 110 & 172 & 180 & 8 \\
\hline Synthetic_10 & 102 & 110 & 182 & 190 & 8 \\
\hline
\end{tabular}

Table 1. Dimensions of synthetic models created for error assessment. All measurements are in mm.

Table 1 provides the dimensions of the ten models used for error assessment. The spherical model was created with inner radius of $102 \mathrm{~mm}$ and outer radius of $110 \mathrm{~mm}$. The top was opened by trimming the model corresponding to a plane offset by $45 \mathrm{~mm}$ from the center of the sphere. For the ellipsoid models, ellipses in the Y-Z plane, with 8 mm between them were revolved around the $\mathrm{Z}$ axis. The same trimming operation was performed to create the opening in the top. Each of these models was used as input to the thickness measurement algorithm outlined in Step 5 shown in the Flow Chart in Figure 4. The thickness measurements for each synthetic model were averaged and compared to the known thickness for each model. The average error rate was compared to the ratio of major-to-minor axis length (from the outer surface) for each model. To approximate major and minor axes lengths for the fat models used in this study, a least-squares ellipsoid fit was performed. This provided a means for comparison to the error assessment data from the synthetic models.

\section{Results}

Two example cardiac fat maps are shown in Figure 7a and 7c, with fat thickness in mm denoted for each coordinate pair of the spherical parameterization of $(\theta, \varphi)$ indicated along the $x$-axis and $y$-axis of the maps, respectively. In addition, the application of the fat thickness to the heart model surface, via interpolated shading, is shown for both example models in Figures $7 \mathrm{~b}$ and $7 \mathrm{~d}$. The mean fat thickness for all 10 models is shown in Figure $8 \mathrm{a}$ and the corresponding heart model with mean fat thickness indicated via interpolated shading is shown in Figure 8b. In addition, the associated standard deviation map and corresponding painted heart model are shown in Figures 8c and 8d, respectively.

In the synthetic models, the average percent error for the spherical model was $1.25 \%$. However, the average percent error for the synthetic model with the longest major axis was 17.3\%. Figure 9 shows a plot of the average percent error versus the ratio of major to minor axis length of the synthetic models, with images of the models included for visual reference. Figure 10 shows an example of an ellipsoid fit to the vertices of one of the fat models. Based on this process, the average major to minor axis ratio was $1.59( \pm 0.24)$. For a ratio of 1.6 , the synthetic models demonstrated an average percent error 
of approximately $14-15 \%$, which, for the $8 \mathrm{~mm}$ thick models, would correspond to an average error of approximately 1.2 $\mathrm{mm}$.
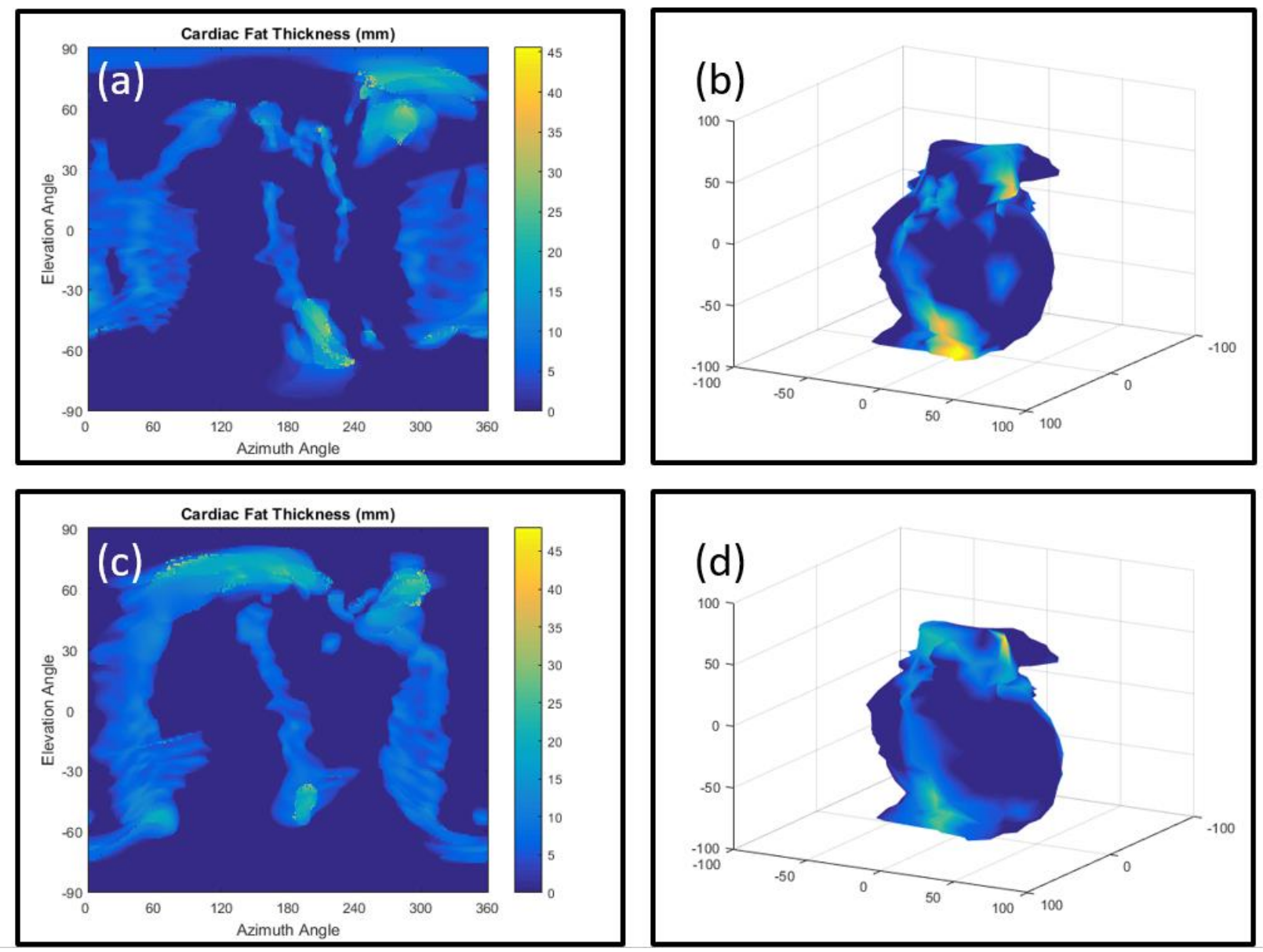

Figure 7. Example results

Example fat maps are shown in (a) and (c) and the corresponding heart surface models with fat thickness applied are shown in (b) and (d), respectively. 

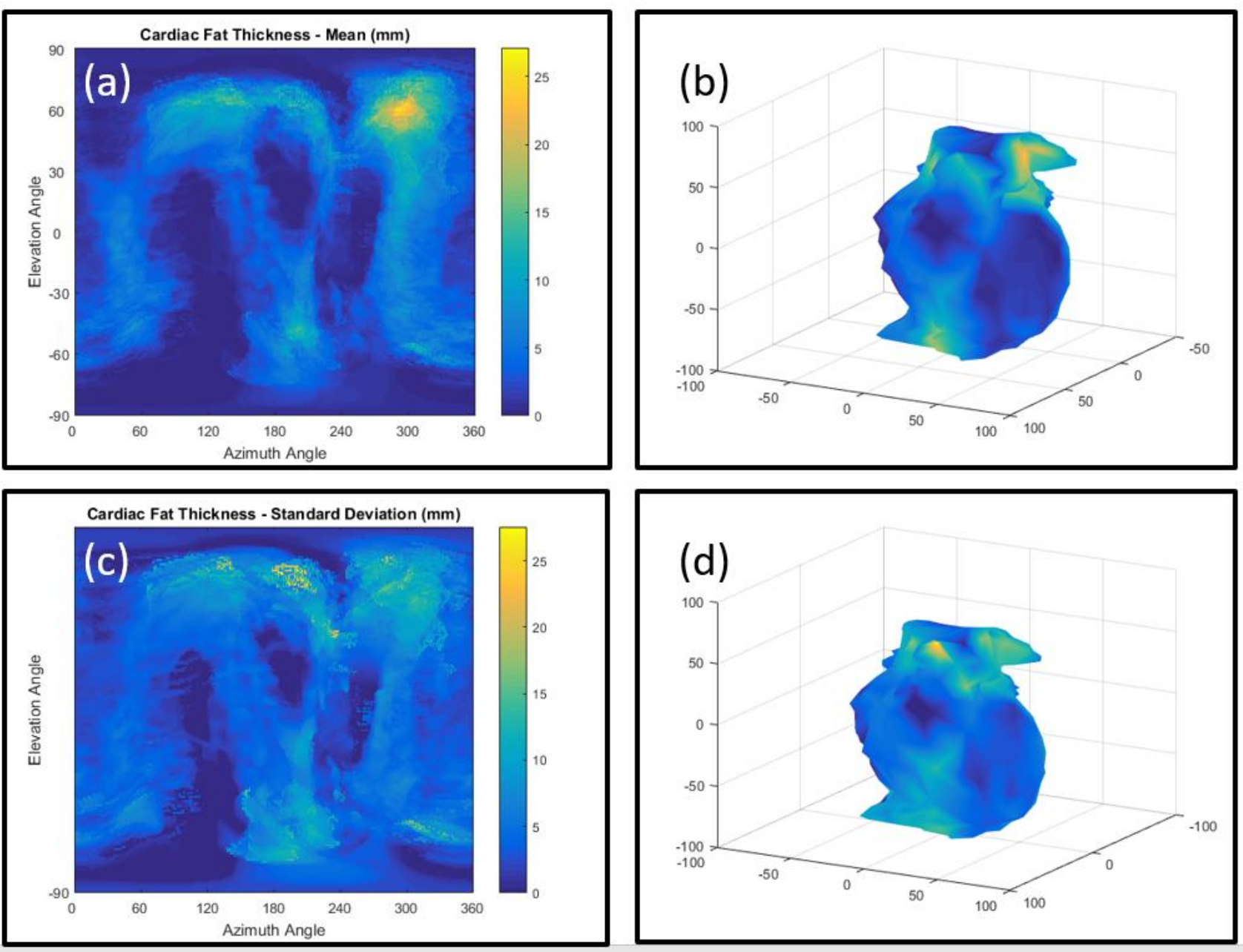

Figure 8. Mean results

Mean fat thickness map (a) and mean heart surface model (b) with fat thickness applied. Also shown are the associated standard deviation map (c) and standard deviation heart surface model (d). 


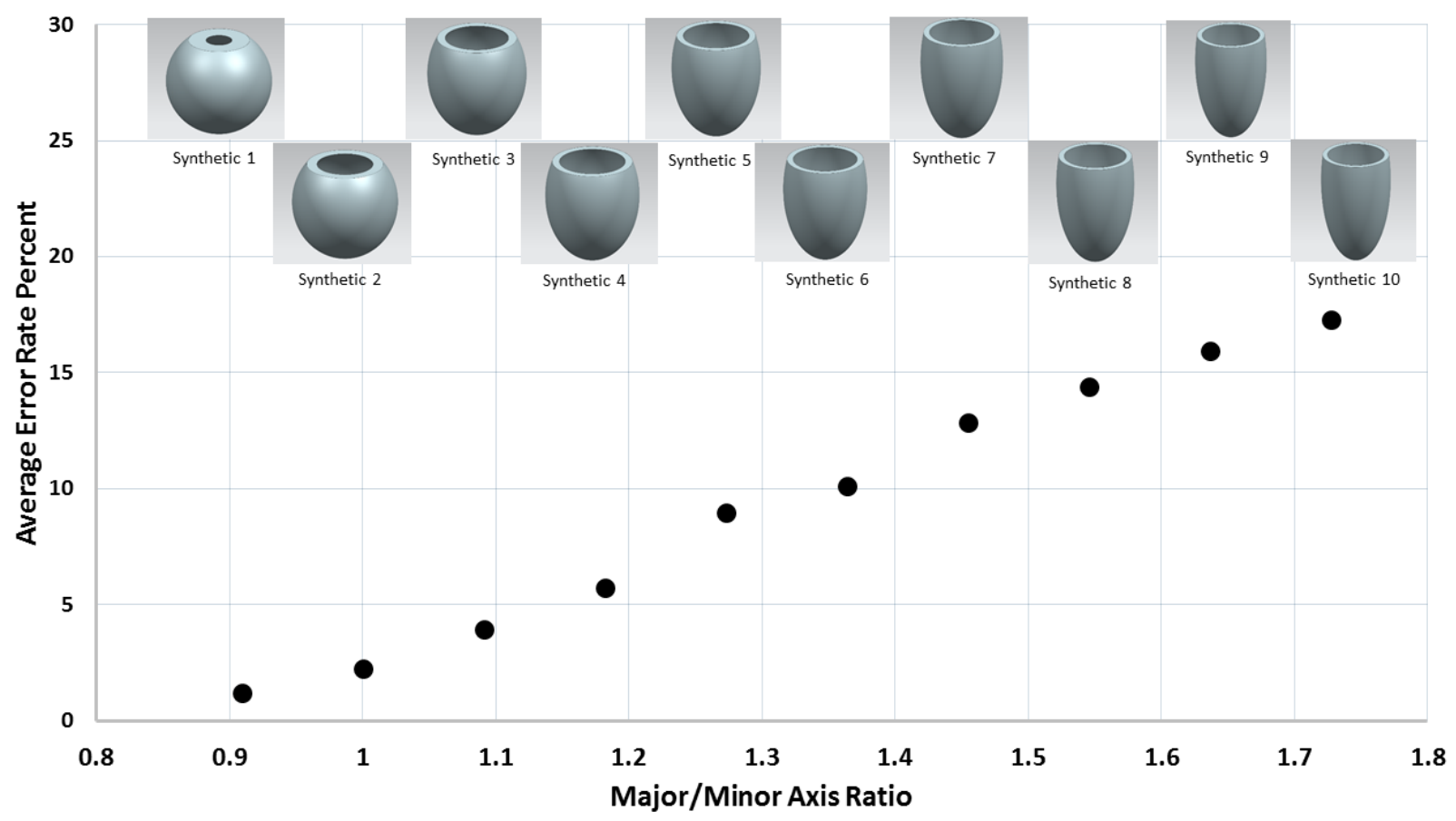

Figure 9. Synthetic models

Plot of average percent error versus major-to-minor axis length ratio for model error assessment. An illustration of each model is included above the corresponding data point for visual reference.

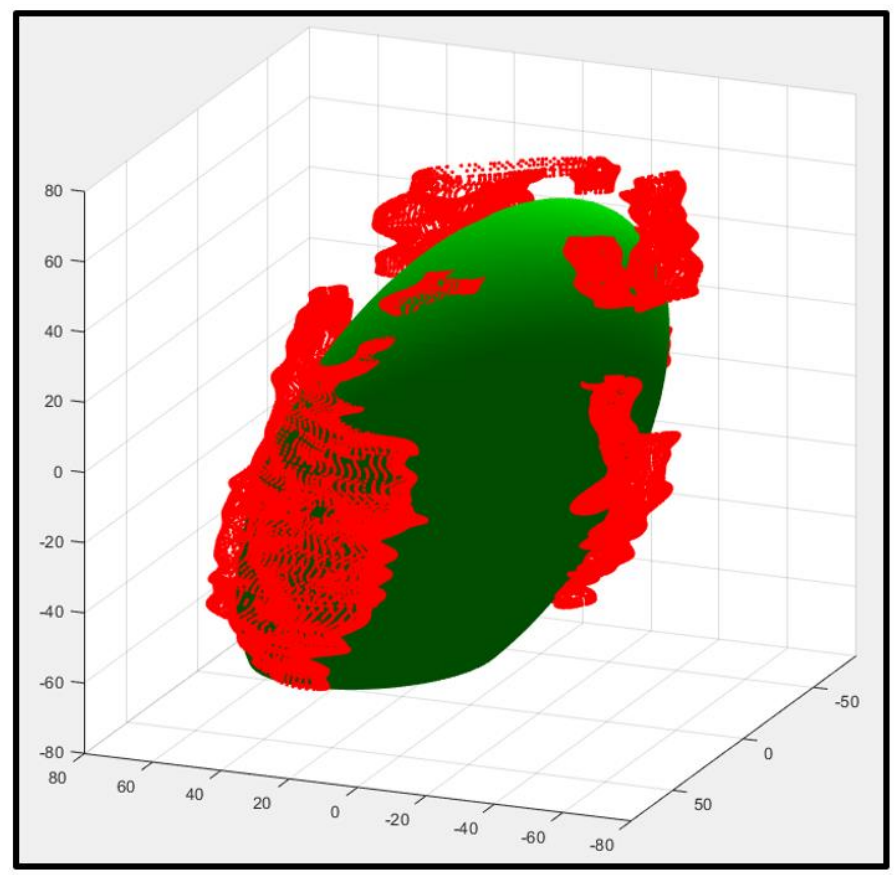

Figure 10. Example ellipsoid fit

Example ellipsoid fit (in green) to an example set of fat model vertices (in red). The resulting major-to-minor axis length ratio was used for comparative assessment with the synthetic model data. 


\section{Discussion}

MRI and CT provide volumetric 3D image data sets that can be used to quantify cardiac fat, but they are expensive imaging modalities and not widely available. Echocardiography, a real-time imaging modality that is substantially less expensive than MRI or CT, has also been used to measure cardiac fat ${ }^{[29]}$. However, typically only single, linear thickness measurements are taken from the free wall of the right ventricle ${ }^{[30,31]}$. Little work assessing the distribution of fat or how it may change with respect to time is available. The model built in this study demonstrates that, while the epicardial and paracardial fat does tend to deposit near the coronary arteries and in the interventricular groove, there is a range of thicknesses and locations of cardiac fat. A SSM built from previously acquired MRI data, incorporating the layer of fat, could be used in conjunction with echocardiography to provide volumetric information without requiring live MRI or CT imaging, overcoming the limitations of single thickness measurements at one location on the right ventricle. An echocardiographic-based system could provide assessment of cardiovascular risk, via the cardiac fat volume and distribution, in a manner substantially less expensive than MRI or CT. In addition, a system like this could be used to study the effect of intervention, including exercise, nutrition, or other treatments, on the distribution of the cardiac fat, without requiring subjects to go to MRI or CT scanners.

The quality of any model is only as good as its input. The Dixon MRI image data sets used as the source of the contours for this study were designed to provide fat quantification for more than just cardiac depots and were not ECG-gated. Therefore, the identification of the epicardial fat (inside the visceral pericardium) separate from the paracardial fat (between the visceral pericardium and the parietal pericardium) was challenging and not attempted for this specific model. They were combined into one depot and labeled cardiac fat. The quantification of the thickness and distribution of cardiac fat in general is not well studied or described in the literature and this model will serve as a foundation to build upon for segmentation algorithm development and for studies in cardiac fat changes with intervention. Additional data can be added to the model, based on cardiac MRI data, as it is available and the model can be evolved to distinguish epicardial from paracardial fat. In addition, the heart and fat contours were extracted from segmented binary images. The segmentation algorithm was described and tested ${ }^{[32]}$, but further validation of its accuracy would lend more confidence to the model.

The model in this study was built using a spherical parameterization. This has several implications - the first of which is related to the typical deposition of the fat. The primary distribution of the fat is across the surface of the myocardium, forming a closed surface with the inner layer of that surface in contact with the myocardium. With this configuration, radial thickness measurements, as derived from the spherical parameterization align well and fit the topology of the surface. However, near the ostia of the coronary arteries, there is often additional fat that appears "inside" the heart when performing the segmentation in this study. Therefore, the simple model of the heart being on the inside and the fat being on the outside does not hold true for the entire surface. This being the case, the approach for making the fat thickness measurements can be thought of as projecting the fat thickness onto the inside of a sphere - areas of the $(\theta, \varphi)$ spherical parameterization where the cast ray intersects additional deposits of fat result in thicker measurements for that location in the map. This effect can be seen in Figure 9a, near elevation angle $80^{\circ}-40^{\circ}$ and azimuth angle $240^{\circ}-300^{\circ}$. It can also be observed on the surface model with fat thicknesses color-coded (Figure 9b). The other artifact of the spherical parameterization is that the "north pole" of the sphere, a single point, maps to the entire first line in the maps of cardiac fat (elevation angle $90^{\circ}$ and azimuth angle $0^{\circ}-360^{\circ}$ ) and the "south pole" maps to the entire last line (elevation angle $-90^{\circ}$ and azimuth angle $0^{\circ}-360^{\circ}$ ). This artifact affects the fat images, but once mapped back onto the heart surfaces, the visibility of the effect is minimized because the mapping is performed in reverse.

An additional consideration of the spherical parameterization is what effect it may have on the approach presented for fat thickness measurement. As expected for a spherical model, the error is small but increases linearly as the model stretches along the major axis, as shown in Figure 9. Based on the ellipsoid fit to the fat models, the average ratio of major to minor axis length was $1.59( \pm 0.24)$. In reference to the plot in Figure 9, this would correspond to approximately $14-15 \%$ error in the fat thickness measurement. While this degree of error is not overwhelming and likely within the resolution of the MRI image data, improvements in the parameterization could reduce the error. In addition, a modified parameterization would allow a modified approach to the direction of the thickness measurement. For example, normals from the heart surface or from the inner surface of the fat model could be calculated and used to provide a direction for thickness computation that is improved from the current spherical approach where rays are cast from one central point.

While the two-dimensional maps of fat thickness alone and the map of mean fat thickness for all 10 models provides information regarding the distribution of cardiac fat, the 3D model required a mean heart surface to act as the substrate for visualization. Many approaches exist for the generation of cardiac models, though most prior work has been focused on the ventricles and the ventricular walls for use in analysis of cardiac function ${ }^{[33]}$. Typically, models are created via matched sets of landmarks from each of the training data sets. Frangi et al. developed models of the left and right ventricle using a 
multi-resolution non-rigid registration technique and a custom approach for automated landmark identification ${ }^{\text {[34] }}$ Lotjonen et al. used a similar approach, but included the atria in their modeling ${ }^{[18]}$. Lorenz and von Berg presented a more comprehensive model and included both the coronary arteries and the large vessels ${ }^{[20]}$. They used a deformation algorithm to match a surface model to the image data to identify surfaces for each image data set and used Procrustes registration to determine a mean surface model. In this study, the myocardial surface models were registered in a similar manner using Procrustes analysis, where point-correspondence was assumed between corresponding vertices of each mesh. The advantage of this approach is that no landmark identification was necessary, as is typical for point-distribution models (PDMs). However, a bias towards the reference mesh can result.

A process was developed, based on Dixon MRI data, to provide 3D models of the myocardial surface and the cardiac fat. Because the fat was identified and included in the modeling process, the models provide a means to assess CAT thickness and distribution on the myocardial surface. The models were aligned into a common frame of reference using an ICP registration algorithm, facilitating the creation of fat thickness maps for each model. The thickness measurements were created by casting rays, computing intersections with the fat surface model, and accumulating thickness. An average heart surface was created using Procrustes analysis and the mean fat thickness map was applied to the average heart surface model for visualization and assessment of fat distribution. Experiments were performed to assess the error resulting from the spherical parameterization used. Synthetic models were created, with successively larger major-to-minor axis length ratios, and used as input to the thickness computation algorithm. The average percent error versus major-to-minor axis ratio was calculated. Ellipsoids were fit to the fat model points to provide an approximate major-to-minor axis ratio for the model data and provide a comparative assessment with the errors derived from the synthetic data. The data from the model error assessment suggests that the process developed in this study provides valid and meaningful information regarding fat thickness and its distribution across the myocardial surface.

The current study is limited in that only 10 MRI scans have been used to create the current model. However, this work demonstrates a framework that can be used moving forward and data from other studies can be added to improve the robustness of the model. Additional validation of the segmentation algorithm would also strengthen the process. Future work will investigate the integration of the model itself into the segmentation strategy to improve the utilization of a priori shape information. The model developed here can be used for both MRI segmentation and as a basis for an ultrasound-based system where volume measurements can be created by registering ultrasound image planes to the 3D model and a deformable model or active surface algorithm is applied based on the CAT identified in the ultrasound planes.

\section{Acknowledgements}

This work was supported by an American Heart Association Institutional Research Enhancement Award (\#17AIREA33670361).

\section{References}

[1] Cornier MA, Després JP, Davis N, Grossniklaus DA, Klein S, Lamarche B, Lopez-Jimenez F, Rao G, St-Onge MP, Towfighi A, Poirier P. Assessing adiposity: A scientific statement from the american heart association. Circulation 2011; 124(18):1996-2019. https://doi.org/10.1161/CIR.0b013e318233bc6a

[2] Van Gaal LF, Mertens IL, De Block CE. Mechanisms linking obesity with cardiovascular disease. Nature 2006; 444(7121):875-80. https://doi.org/10.1038/nature05487

[3] Iacobellis G, Corradi D, Sharma AM. Epicardial adipose tissue: Anatomic, biomolecular and clinical relationships with the heart. Nat Clin Pract Cardiovasc Med 2005; 2(10):536-43. https://doi.org/10.1038/ncpcardio0319

[4] Talman AH, Psaltis PJ, Cameron JD, Meredith IT, Seneviratne SK, Wong DT. Epicardial adipose tissue: Far more than a fat depot. Cardiovasc Diagn Ther 2014; 4(6):416-29. https://doi.org/10.3978/j.issn.2223-3652.2014.11.05

[5] Alexopoulos N, McLean DS, Janik M, Arepalli CD, Stillman AE, Raggi P. Epicardial adipose tissue and coronary artery plaque characteristics. Atherosclerosis 2010; 210(1):150-4. https://doi.org/10.1016/j.atherosclerosis.2009.11.020

[6] Cullu N, Kantarci M, Kizrak Y, Pirimoglu B, Bayraktutan U, Ogul H, Karaca L. Does epicardial adipose tissue volume provide information about the presence and localization of coronary artery disease? Anatol J Cardiol 2015; 15(5):355-9. https://doi.org/10.5152/akd.2014.5431

[7] Nakazato R, Shmilovich H, Tamarappoo BK, Cheng VY, Slomka PJ, Berman DS, Dey D. Interscan reproducibility of computer-aided epicardial and thoracic fat measurement from noncontrast cardiac CT. J Cardiovasc Comput Tomogr 2011; 5(3):172-9. https://doi.org/10.1016/j.jcct.2011.03.009

[8] Nikolaou K, Flohr T, Knez A, Rist C, Wintersperger B, Johnson T, Reiser MF, Becker CR. Advances in cardiac CT imaging: 64-slice scanner. Int J Cardiovasc Imaging 2004; 20(6):535-40. https://doi.org/10.1007/s10554-004-7015-1 
[9] Mihl C, Loeffen D, Versteylen MO, Takx RAP, Nelemans PJ, Nijssen EC, Vega-Higuera F, Wildberger JE, Das M. Automated quantification of epicardial adipose tissue (EAT) in coronary CT angiography; comparison with manual assessment and correlation with coronary artery disease. J Cardiovasc Comput Tomogr 2014; 8(3):215-21. https://doi.org/10.1016/j.jcct.2014.04.003

[10] Rodrigues EO, Conci A, Morais FFC, Pérez MG. Towards the automated segmentation of epicardial and mediastinal fats: A multi-manufacturer approach using intersubject registration and random forest. IEEE International Conference on Industrial Technology (ICIT) 2015:1779-85. https://doi.org/10.1109/ICIT.2015.7125355

[11] Spearman JV, Meinel FG, Schoepf UJ, Apfaltrer P, Silverman JR, Krazinski AW, Canstein C, De Cecco CN, Costello P, Geyer LL. Automated quantification of epicardial adipose tissue using CT angiography: Evaluation of a prototype software. Eur Radiol 2014; 24(2):519-26. https://doi.org/10.1007/s00330-013-3052-2

[12] Thomas EL, Fitzpatrick JA, Malik SJ, Taylor-Robinson SD, Bell JD. Whole body fat: Content and distribution. Prog Nucl Magn Reson Spectrosc 2013; 73:56-80. https://doi.org/10.1016/j.pnmrs.2013.04.001

[13] Dixon WT. Simple proton spectroscopic imaging. Radiology 1984; 153(1):189-94. https://doi.org/10.1148/radiology.153.1.6089263

[14] Bonekamp S, Ghosh P, Crawford S, Solga S, Horska A, Brancati F, Diehl A, Smith S, Clark J. Quantitative comparison and evaluation of software packages for assessment of abdominal adipose tissue distribution by magnetic resonance imaging. Int $\mathrm{J}$ Obes (Lond) 2008; 32(1):100-11. https://doi.org/10.1038/sj.ijo.0803696

[15] Cristobal-Huerta A, Torrado-Carvajal A, Malpica N, Luaces M, Hernandez-Tamames JA. Automated quantification of epicardial adipose tissue in cardiac magnetic resonance imaging. International Conference of the IEEE Engineering in Medicine and Biology Society (EMBC) 2015:7308-11. https://doi.org/10.1109/EMBC.2015.7320079

[16] Homsi R, Meier-Schroers M, Gieseke J, Dabir D, Luetkens JA, Kuetting DL, Naehle CP, Marx C, Schild HH, Thomas DK, Sprinkart AM. 3D-dixon MRI based volumetry of peri- and epicardial fat. Int J Cardiovasc Imaging 2016; 32(2):291-9. https://doi.org/10.1007/s10554-015-0778-8

[17] Klingensmith JD, Elliott AL, Fernandez-del-Valle M, Mitra S. Automated segmentation of cardiac adipose tissue in dixon magnetic resonance images. J Biomed Graph Comput; Forthcoming 2018. https://doi.org/10.5430/jbgc.v8n1p1

[18] Lötjönen J, Kivistö S, Koikkalainen J, Smutek D, Lauerma K. Statistical shape model of atria, ventricles and epicardium from short- and long-axis MR images. Med Image Anal 2004; 8(3):371-86. https://doi.org/10.1016/j.media.2004.06.013

[19] Lorenzo-Valdés M, Sanchez-Ortiz GI, Elkington AG, Mohiaddin RH, Rueckert D. Segmentation of 4D cardiac MR images using a probabilistic atlas and the EM algorithm. Med Image Anal 2004; 8(3):255-65. https://doi.org/10.1016/j.media.2004.06.005

[20] Lorenz C, von Berg J. A comprehensive shape model of the heart. Med Image Anal 2006; 10(4):657-70. https://doi.org/10.1016/j.media.2006.03.004

[21] Albà X, Pereañez M, Hoogendoorn C, Swift AJ, Wild JM, Frangi AF, Lekadir K. An algorithm for the segmentation of highly abnormal hearts using a generic statistical shape model. IEEE Trans Med Imaging 2016; 35(3):845-59. https://doi.org/10.1109/TMI.2015.2497906

[22] Ardekani S, Jain S, Sanzi A, Corona-Villalobos CP, Abraham TP, Abraham MR, Zimmerman SL, Wu KC, Winslow RL, Miller MI, Younes L. Shape analysis of hypertrophic and hypertensive heart disease using MRI-based 3D surface models of left ventricular geometry. Med Image Anal 2016; 29:12-23. https://doi.org/10.1016/j.media.2015.11.004

[23] Wile GE, Leyendecker JR. Magnetic resonance imaging of the liver: Sequence optimization and artifacts. Magn Reson Imaging Clin N Am 2010; 18(3):525-47. https://doi.org/10.1016/j.mric.2010.07.010

[24] Vogt FM, Antoch G, Hunold P, Maderwald S, Ladd ME, Debatin JF, Ruehm SG. Parallel acquisition techniques for accelerated volumetric interpolated breath-hold examination magnetic resonance imaging of the upper abdomen: Assessment of image quality and lesion conspicuity. J Magn Reson Imaging 2005; 21(4):376-82. https://doi.org/10.1002/jmri.20288

[25] Gonzalez, RC, Eddins, S, Woods, RE. Digital image processing using MATLAB. [Internet]. Upper Saddle River, NJ: Pearson / Prentice Hall; 2004

[26] Gower, JC, Dijksterhuis, GB. Procrustes problems. [Internet]. Oxford University Press on Demand; 2004

[27] Bergström P, Edlund O. Robust registration of point sets using iteratively reweighted least squares. Comput Optim Appl 2014; 58(3):543-61. https://doi.org/10.1007/s10589-014-9643-2

[28] Moller T, Trumbore B. Fast, minimum storage ray-triangle intersection. J Graph Tools 1997; 2(1):21-8. https://doi.org/10.1080/10867651.1997.10487468

[29] Iacobellis G, Ribaudo MC, Assael F, Vecci E, Tiberti C, Zappaterreno A, Di Mario U, Leonetti F. Echocardiographic epicardial adipose tissue is related to anthropometric and clinical parameters of metabolic syndrome: A new indicator of cardiovascular risk. J Clin Endocrinol Metab 2003; 88(11):5163-8. https://doi.org/10.1210/jc.2003-030698 
[30] Silaghi A, Piercecchi-Marti MD, Grino M, Leonetti G, Alessi MC, Clement K, Dadoun F, Dutour A. Epicardial adipose tissue extent: Relationship with age, body fat distribution, and coronaropathy. Obesity 2008; 16(11):2424-30. https://doi.org/10.1038/oby.2008.379

[31] Iacobellis G, Assael F, Ribaudo MC, Zappaterreno A, Alessi G, Di Mario U, Leonetti F. Epicardial fat from echocardiography: A new method for visceral adipose tissue prediction. Obes Res 2003; 11(2):304-10. https://doi.org/10.1038/oby.2003.45

[32] Hill JE, Fernandez-Del-Valle M, Mitra S. An automated segmentation for direct assessment of adipose tissue distribution from thoracic and abdominal multi-echo dixon MR images. SPIE Medical Imaging 2017; 10133-15:1-11. https://doi.org/10.1117/12.2254481

[33] Frangi AF, Niessen WJ, Viergever MA. Three-dimensional modeling for functional analysis of cardiac images, a review. IEEE Trans Med Imaging 2001; 20(1):2-5. https://doi.org/10.1109/42.906421

[34] Frangi AF, Rueckert D, Schnabel JA, Niessen WJ. Automatic construction of multiple-object three-dimensional statistical shape models: Application to cardiac modeling. IEEE Trans Med Imaging 2002; 21(9):1151-66. https://doi.org/10.1109/TMI.2002.804426 